\title{
orobaNCHE BALLOTAE A. PUJADAS (OROBANCHACEAE), ESPECIE NUEVA
}

\author{
Antonio J. PUJADAS SALVÀ
}

\begin{abstract}
RESUMEN. Orobanche ballotae A. Pujadas (Orobanchaceae), especie. Se describe para el $\mathrm{S}$ de la Península Ibérica una especie nueva del género Orobanche L., de la sección Orobanche, Grex Minores G. Beck. Se destacan sus caracteres morfológicos que permiten diferenciarlo claramente de O. minor Sm., especie con la que presenta ciertas semejanzas.
\end{abstract}

Palabras clave. Orobanchaceae, Orobanche, jopo, parásito.

ABSTRACT. Orobanche ballotae A. Pujadas (Orobanchaceae), a new species. A new species of the genus Orobanche L. that belongs to the Section Orobanche, Grex Minores G. Beck is described from the south of the Iberian Peninsula. Its morphological characteristics are pointed out; the differences with $O$. minor Sm., a close allied, are also analyzed.

Key words. Orobanchaceae, Orobanche, broomrape, parasite.

\section{INTRODUCCIÓN}

La Región Mediterránea constituye un importante centro de diversidad del género Orobanche y sobre todo para la Grex Minores G. Beck (Beck 1890). Son los trabajos de Beck (1890 y 1930) los que, principalmente, han aportado un mayor conocimiento sobre la taxonomía, nomenclatura y corología de este grupo, otros autores como Guimaraes (1904) y Rumsey \& Jury (1991) también han realizado diferentes contribuciones sobre el mismo grupo a nivel local o parcial. A pesar de estas obras aun siguen existiendo problemas sobre su tratamiento taxonómico, nomenclatural y en muchos casos sobre el conocimiento de su área de distribución.

En este trabajo damos a conocer una nueva especie para el sur de la Península Ibérica incluible en la Grex Minores G. Beck, Sect. Orobanche. Especie que en anteriores estudios habíamos incluído en O. minor Sm. (Pujadas Salvà et al.1994) o considerado simplemente como una variante albina del mismo y que llevados por nuestro interés en subordinarlo a un taxón ya existente lo denominamos de forma errónea como O. minor fma. albens G. Beck (Pujadas Salvà \& Lora González 1996). Éste sería el único caso posible, dentro de la Grex Minores, que presenta el estigma 
completamente blanco, según la literatura consultada. Su asignación a cualquier otro taxa del género no ha sido posible.

Aportamos su diagnosis, descripción, ecología y un icon original. Se compara con otros ejemplares típicos de O. minor, recolectados en Andalucía, para su reconocimiento como nueva especie.

\section{MATERIAL Y MÉTODO}

Las observaciones se han realizado a partir de planta viva y de especímenes de herbario. Los ejemplares recolectados se encuentran depositados en el Herbario COA, además se han revisado los siguientes herbarios nacionales y regionales $\mathrm{ABH}, \mathrm{ALME}, \mathrm{BC}, \mathrm{BCC}, \mathrm{BCF}$, GDA, GDF, MA, MGC, MUB, SEV, VAB y VAL.

Se comparan los caracteres morfológicos del nuevo taxón con los de ejemplares característicos de $O$. minor $\mathrm{Sm}$. var. minor de Andalucía. No se ha podido localizar, para su análisis, el material tipo correspondiente a $O$. minor fma. albens, en ninguno de los herbarios consultados donde con mayor probabilidad podría haberse encontrado (B, BP, G, GENT, PR, PRC, TUB, W).

Para el estudio taxonómico y nomenclatural de los diferentes especímenes nos hemos basado principalmente en los trabajos de Beck (1890 y 1930), Guimaraes (1904), Chater \& Webb (1972) y Rumsey \& Jury (1991).

\section{RESULTADOS}

Orobanche ballotae A. Pujadas, sp. nov. =O. minor fma. albens sensu A. Pujadas \& A. Lora in Acta Bot. Malacitana 21: 215 (1996), non Beck in Repert. Spec. Nov. Regni Veg. Beih. 18: 40 (1922)

Caulis gracilis, albus, vel dilute purpureus, basi parum incrassatus. Spica cylindrata, basi conspicue vel tota laxiflora. Bracteae corollam longitudine superantes, interdum aequantes, apice saepe deflexo. Calyx $5-9$ mm longus; partes anticae contiguae vel imbricatae, basi ample ovales, ex subintegris usque ad inaequalissime bidentatas, dentibus subulatis acuminatis, corollae tubum breviores vel subaequantes, albus. Corolla 10-14(15) mm longa, tubulosa, base parum inflata, limbus vix galeatus, primo erecta vel erectopatens demum patens, post anthesim constricta et prona supra germen, saepe genuflexa, albalactea, interdum nervis limbi tenuiter amethystinis. Filamenta postica 2-3,5 mm supra basim corollae oblique inserta, filamenta antica (1)1,5-2 $\mathrm{mm}$ horizontaliter inserta; filamenta basi dilatata, infra usque ad 2/3 partem pilis longis usque $0,5(1) \mathrm{mm}$, apice pilis glanduliferis perpaucis obsito, alba cum basi lutea. Antherae 1,5-1,8 $\mathrm{mm}$ longae, in sutura base puberulae, amethystinae, albaemucronulatae. Germen breviter et parce glandulosum pilosum acuminatum, album basi lutea. Stylus parce glandulosus-pilosus, albus interdum dilute amethystinus. Stigma bilobum, album-lacteum. Plantae nutriens: Ballota hirsuta Bentham.

Derivatio nominis: "ballotae" por haber sido encontrado parasitando exclusivamente la Ballota hirsuta Bentham.

Typus. CÓRDOBA: Almodóvar, base del Castillo, 30SUG2186, 200msm, sobre Ballota hirsuta, 2-V-1991, A. Pujadas, P. Poyato, A. Segui y A. Ramírez, COA 17617, holotypus. (Isotypus: G, MA, MGC).

\section{Iconografía: Figura 1.}

Descripción. Escapo (16)22-34(43) cm de alto, simple a veces formando agregados, tallo de (3)4-6(7) mm de diámetro en su mitad, de 5-8(12) mm apenas engrosado en su base, 


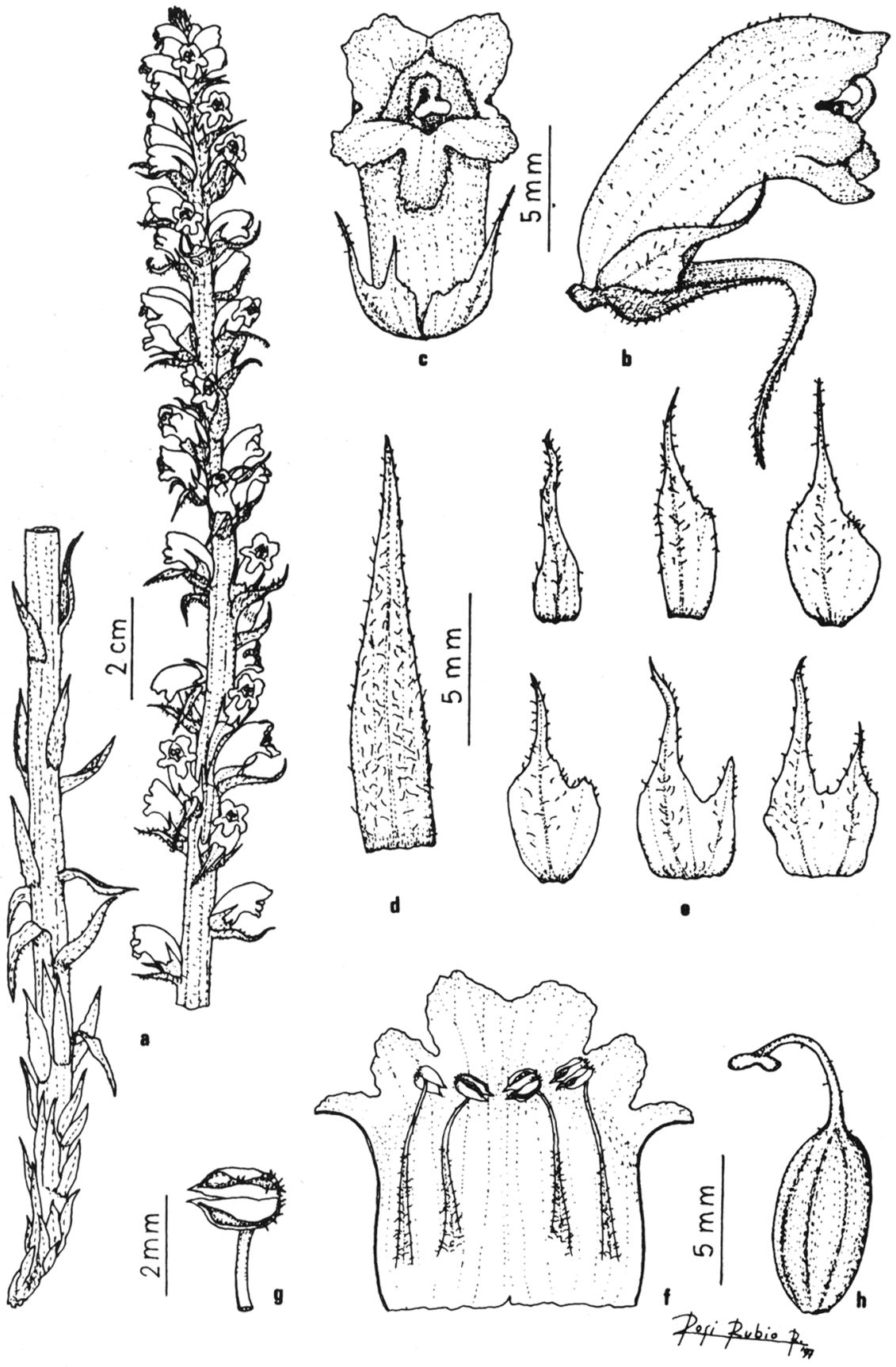

Figura 1. Orobanche ballotae: a) hábito; b) flor, visión lateral; c) flor, visión frontal; d) bráctea; e) segmentos del cáliz, variabilidad; f) corola abierta y androceo; g) antera; h) gineceo. Orobanche ballotae: a) appearance; b) flower, side view; c) flower, front view, d) bract; e) segments of the calyx, variability, f) open corolla and androecium; g) anther; h) gynoecium. 
subtetrágono y ligeramente estriado, pubescente glandular, blanquecino a veces ligeramente teñido de púrpura, pardo rojizo en seco. Hojas basales de 10-15 x4-5 mm, ovado lanceoladas, subimbricadas, glabras, las superiores 15-19(21) x 2-3(4) mm, gradualmente más largas, estrechamente lanceoladas, espaciándose en altura, erectopatentes, blancas, a veces ligeramente teñidas de violeta que se tornan rápidamente de color pardo, pubescentes glandulares en su lado abaxial. Espiga (10)13-19(28) x 2-2,8 cm, cilíndrica más o menos redondeada en el ápice, escasamente comosa, multiflora, laxa al menos en su mitad inferior. Flores erectas a erecto patentes. Brácteas (11)14-17 mm mayores o igualando la corola, semejantes a las hojas, frecuentemente reflejas. Cáliz de 5-9 mm, menor que el tubo de la corola, pelosoglandular, blanco a veces con su ápice ligeramente violáceo, sin nerviación conspicua; segmentos libres, contiguos o brevemente imbricados raramente soldados en la base, subenteros a muy desigualmente bidentados (generalmente los inferiores muy desigualmente bidentados y los superiores subenteros), ampliamente ovados en su base (1.5)2,5-3,5 mm de ancho- y dientes subulados largamente acuminados. Corola 10-14(15) mm de longitud, 4-5 mm de di.metro en su mitad, tubulosa algo hinchada en la base y ligeramente galeada en el limbo, uniforme y marcadamente curvada, erecta a erecto patente, en la fructificación recurvada, genuflexa y constreñida por encima del ovario, exteriormente pubescente glandular con pubescencia mas densa hacia el limbo y glabra o glabrescente en su vientre, interiormente pelosilla en su base, blanco lechosa, raramente los nervios de los lóbulos ligeramente ametisteos. Labio superior bilobado escasamente emarginado, porrecto, el inferior con el lóbulo medio mayor que los laterales, irregularmente dentados no ciliados. Estambres 4; los filamentos adaxiales de 6-7 $\mathrm{mm}$ de longitud insertos oblicuamente a 2-3,5 mm de la base de la corola, los abaxiales de $7-8 \mathrm{~mm}$ insertos horizontalmente a (1) 1,5-2 mm, dilatados inferiormente de $(0,5)$ 0,7-0,8(1,1) mm de ancho, todos con pelos largos de hasta 0,5 (1) $\mathrm{mm}$ en su base (hasta los 2/3 inferiores de su longitud) y ápice glabrescente con escasos pelos glandulares cortos, esparcidos, poco conspicuos, filamentos blancos con la base amarilla. Anteras 1,5-1,8 mm, elipsoideas, de bases redondeadas con apículo corto de 0,1$0,2(0,3) \mathrm{mm}$ blanquecino, pubérulas en la base de la sutura, ametisteas (gris-ametisteo en seco). Ovario 7,5-8(9) $\mathrm{mm}$ glabrescente con escasos pelos glandulares en el lado abaxial de su ápice, blanco, amarillo en su base. Estilo de 6-7 mm, con pelos glandulares esparcidos a veces glabrescente, blanco o ligeramente teñido de ametistea en su ápice. Estigma bilobado, blanco lechoso. Semillas 0,3-0,5 x 0,2-0,25 $\mathrm{mm}$, de piriformes, subcilíndricas a subesféricas, gris negruzcas. Parasita sobre Ballota hirsuta Bentham.

Ecología. Habita en zona de matorrales xerofíticos degradados del piso termomediterráneo en lugares ruderalizados 0 subruderalizados parasitando Ballota hirsuta Bentham. Se encuentra entre 20 y $1500 \mathrm{msm}$. Florece de Abril a Mayo, fructifica de Mayo a Junio.

Distribución. Se ha localizado en Andalucía, por ahora en las provincias de Almería, Córdoba, Granada, Jaén y Málaga . Su presencia es muy probable en toda la mitad sur peninsular y en el noroeste de África.

\section{DISCUSIÓN Y CONCLUSIÓN}

En todas las poblaciones observadas de Córdoba y Almería hemos podido verificar la constancia de los caracteres que diferencian $O$. ballotae de 0 . minor (tab. 1) como son el color blaquecino de toda la planta, los segmentos del cáliz menores y más anchos, corola de tamaño 


\begin{tabular}{|c|c|c|}
\hline & O. minor & O. ballotae \\
\hline Tallo & marrón o ametisteo & blanquecino \\
\hline Cáliz longitud (mm) & (8) $10-16(18)$ & $5-9$ \\
\hline \multicolumn{3}{|l|}{ Cáliz, anchura de la base de } \\
\hline los segmentos $(\mathrm{mm})$ & $1-3$ & $(1,5) 2,5-3,5$ \\
\hline Cáliz, dientes & lanceolados & subulados \\
\hline Cáliz, división de los segmentos & $\begin{array}{l}\text { Ápice subfiliforme } \\
\text { subenteros a ligeramente desigual y } \\
\text { profundamente bidentados }\end{array}$ & $\begin{array}{l}\text { Ápicelargamenteacuminado } \\
\text { subenteros a muy } \\
\text { desigualmente bidentados }\end{array}$ \\
\hline Cáliz, dientes & divergentes & convergentes o paralelos \\
\hline Corola, longitud (mm) & $13-17$ & $10-14(15)$ \\
\hline Corola, diámetro (mm) & $4-6$ & $4-5$ \\
\hline Corola, forma de la base & ligeramente curvada & marcadamente curvada \\
\hline Corola, forma del dorso & $+/-$ recto & curvo \\
\hline Corola, labios & divergentes $+/$ - patentes & porrectos \\
\hline Corola, color & blanco profundamente teñido de púrpura & blanco \\
\hline Anteras, longitud (mm) & $1,1-1,3(1,5)$ & $1,5-1,8$ \\
\hline Anteras, forma & ovoideas & elipsoideas \\
\hline Anteras, indumento en su base & glabras o con tricomas papilosos & pubérulas \\
\hline Estigma & amarillo(inicialmente) a purpúreo & blanco lechoso \\
\hline
\end{tabular}

Tabla 1. Caracteres taxonómicos de interés para la delimitación de $O$. minor y $O$. ballotae. Taxonomic characters in the identification of $O$. minor and O. ballotae.

menor y más curvada, anteras de mayor longitud, elipsoideas, siempre pubérulas en la base de la sutura y el estigma de color blanco lechoso. Además se han estudiado dos poblaciones, de Málaga y Jaén, recolectadas en estado muy avanzado (casi secas) y un pliego de Granada del Herbario GDA que conserva dos especímenes aparentemente parasitando a Ballota hirsuta (no se indica expresamente en la etiqueta de herbario pero aparece un fragmento de esta especie junto al Orobanche); aunque en estos casos no se ha podido tener en cuenta el color de las diferentes partes de la planta para su identificación, como carácter más llamativo, sin embargo la constancia de los demás caracteres morfológicos como son forma y tamaño de las brácteas, de los segmentos del cáliz, de la corola y de las anteras nos ha permitido incluir a los individuos estudiados bajo el mismo taxón y constatar la clara separación entre $O$. ballotae y $O$. minor

A pesar de no haber sido posible localizar el material tipo de $O$. minor fma. albens para su estudio, creemos que la diagnosis realizada por Beck (1922) aporta suficiente información para diferenciarlo de $O$. ballota.

El hecho de haber sido recolectado en diferentes localidades de Andalucía muy separadas entre sí como son Córdoba y Almería y de presentar casi toda la planta un color blanco-lechoso, el estigma constantemente de color blanco, el de ser parásito exclusivo de Ballota hirsuta además de otros caracteres morfológicos diferenciales de la flor (tab. 1), nos permite reconocerlo como un nuevo taxón a nivel específico, descartando su consideración de variedad o de mera forma albina a nivel local subordinada a $O$. minor. 


\section{MATERIAL ESTUDIADO}

\section{O. ballotae A. Pujadas}

ALMERÍA: Cerro Majada Redonda, Níjar, 30SWF8176, 400 m, sobre Ballota hirsuta, 13-IV1994, A. Pujadas, COA 17444. Norte del barranco del Sabinar, cortijo El Rincón de Martos, Níjar, 30SWF7470, $350 \mathrm{~m}$, sobre Ballota hirsuta, 19-IV1994, A. Pujadas y R. Jiménez,19-IV-1994, COA 17447. Cerro de Los Frailes, Níjar, 30SWF8171, 300m, sobre Ballota hirsuta, 2-V-1994, A. Pujadas, COA 17446. Cerro del Garbanzal, Níjar, 30SWF8176, $200 \mathrm{~m}$, sobre Ballota hirsuta, 6-V1994, A. Pujadas, COA 17445. Agua Amarga, Níjar, 30SWF9588, $20 \mathrm{~m}$, sobre Ballota hirsuta, 6-V1994, A. Pujadas, COA 17427. Entre Presillas Bajas y cerro de La Majada Redonda, Níjar, 30SWF8176, 200 m, sobre Ballota hirsuta, 8-IV-1995, A. Pujadas, COA 17448. CÓRDOBA: Almodóvar, base del Castillo, 30SUG2186, $200 \mathrm{~m}$, sobre Ballota hirsuta, 2-V-1991, A. Pujadas, P. Poyato, A. Segui \& A. Ramírez, COA 17617 (holotypus). GRANADA: Cogollos de Vega, Peñón de la Mata, vertiente N, 30SVG5227, 1500 m, 22-V-1988, O. Socorro, L. Cano \& M.C. Espinar, GDA 22367. JAÉN: Los Cañones, Los Villares, 30SVG3073, 560 m, 12-VI1991, A. Pujadas \& A. Lora, COA 17618. MÁLAGA: Marchamonas, Periana, 30SUF9591, 900 m, 24-VI1992, A. Pujadas, A. Lora \& P. Prados, COA 22224.

\section{O. minor $\mathrm{Sm}$.}

CÁDIZ: Los Barrios, C-440 Km 68, sobre Ononis sp., 11-V-1988, J. Oliveira, COA 13535. Finca El Zapatero, Benalup, 30STF6023, 300 m, sobre Trifolium subterraneum L., 4-V-1995, A. Pujadas y A. Lora, COA 17620. CÓRDOBA: De Obejo a Villanueva Km 10, 30SUH4624, 520 m, 26IV-1983, A. Pujadas, COA 13519. De Cardeña a Montoro Km 25, 30SUH8717, 350 m, 22-IV-1988, A. Pujadas, COA 13536. De Córdoba a Villaviciosa Km 6, 30SUH3900, 570 m, 1-X-1988, A. Pujadas y P. Poyato, COA 13541. Arroyo de la Corcobada, Cardeña, 30SUH8631, 720 m, 24-V-1989, A. Pujadas y J. Oliveira, COA 13494. De Cardeña a Villa del Río Km 10, 30SUH8826, 740 m, 24-V1989, A. Pujadas y J. Oliveira, COA 13516. Puente Genil, sobre Trifolium tomentosum L., 25-V-1989, J. Oliveira, COA 1387I. Villaviciosa, alrededores, 30SUH2415, $700 \mathrm{~m}, 4-\mathrm{VI}-1989$, A. Pujadas y $P$. Poyato, COA 13517.
AGRADECIMIENTOS. Agradecemos a D. José Santos Cabello Pérez la versión latina de la diagnosis y a los conservadores de los herbarios ABH, ALME, BC, BP, G, GDA, GDAC, MGC, MUB, SEV, TUB, VAB, VAL y W, las facilidades dadas en las consultas de sus respectivos herbarios.

\section{BIBLIOGRAFÍA}

BECK, G. -1890- Monogaphie der Gattung Orobanche. Bibliotheca Botanica 19: 1-275.

BECK, G. -1922-Orobancheae novae. Repert. Spec. Nov. Regni Veg. Beih. 18: 33-40.

BECK, G. -1930- Orobanchaceae. In: Engler (de.), Pflanzenreich 96 (IV.261): 1-348.

CHATER, A.D. \& D.A. WEBB -1972- Orobanche. In: T.G. Tutinet al.(eds.), Fl. Europ. vol. 3: 286-293. Cambridge.

GUIMARAES, J.A. -1904- Monografía das Orobanchaceas. Broteria 3: 1-208.

PUJADAS SALVÀ, A., E. HERNÁNDEZ BERMEJO \& J.A.R. OLIVEIRA VELLOSO 1994- The Genus Orobanche in Andalusia (southern Spain): Taxonomical, chorological \& ecological aspects, in: Pieterse, Verklej \& Ter Borj (Eds.), Biology and management of Orobanche 132-137. Amsterdam.

PUJADAS SALVÀ, A. y A. LORA GONZÁLEZ 1996- El género Orobanche L. (Orobanchaceae) en la provincia de Almería, SE de España. Acta Bot. Malacitana 21: 199-220.

RUMSEY, F.J. \& S.L. JURY - 1991- An account of Orobanche L. in Britain and Ireland. Watsonia 18:257-295.

Aceptado para su publicación en Octubre de 1997

Dirección del autor. Departamento de Ciencias y Recursos Agrícolas y Forestales. Escuela Técnica Superior de Ingenieros Agrónomos y de Montes. Universidad de Córdoba. Apdo. 3048. E-14080 Córdoba. 